\title{
The relationship of genetic susceptibilities for psychosis with physiological fluctuation in functional MRI data
}

\section{Saarinen, Aino}

2020-03-30

Saarinen , A , Lieslehto , J , Kiviniemi , V , Tuovinen , T , Veijola , J \& Hintsanen , M 2020 , '

The relationship of genetic susceptibilities for psychosis with physiological fluctuation in functional MRI data ' , Psychiatry Research. Neuroimaging , vol. 297 , 111031 . https://doi.org/10.1016/j.pscychresns

http://hdl.handle.net/10138/326112

https://doi.org/10.1016/j.pscychresns.2020.111031

cc_by_nc_nd

acceptedVersion

Downloaded from Helda, University of Helsinki institutional repository.

This is an electronic reprint of the original article.

This reprint may differ from the original in pagination and typographic detail.

Please cite the original version. 


\title{
The relationship of genetic susceptibilities for psychosis with physiological fluctuation in functional MRI data
}

\author{
Aino Saarinen ${ }^{1,2,3}$, Johannes Lieslehto ${ }^{3}$, Vesa Kiviniemi ${ }^{4,5}$, Timo Tuovinen ${ }^{3}$, \\ Juha Veijola ${ }^{3,5,6, *}$, Mirka Hintsanen ${ }^{1, *}$
}

${ }^{1}$ Research Unit of Psychology, University of Oulu, Finland

${ }^{2}$ Department of Psychology and Logopedics, Faculty of Medicine, University of Helsinki, Finland

${ }^{3}$ Research Unit of Clinical Neuroscience, Department of Psychiatry, University of Oulu

${ }^{4}$ Department of Diagnostic Radiology, Oulu University Hospital, Oulu, Finland

${ }^{5}$ Department of Psychiatry, Oulu University Hospital, Oulu, Finland

${ }^{6}$ Medical Research Center Oulu, Oulu University Hospital and University of Oulu, Oulu, Finland

* These authors contributed equally.

Corresponding author: Aino Saarinen. Department of Psychology and Logopedics, Faculty of Medicine, Haartmaninkatu 3, P.O. Box 21, 00014 University of Helsinki, Finland. E-mail: aino.i.saarinen@ @elsinki.fi, Tel.: +35844 3071204. 


\begin{abstract}
Previously, schizophrenia is found to be related to the variability of the functional magnetic resonance imaging (fMRI) signal in the white matter. However, evidence about the relationship between genetic vulnerability for schizophrenia and physiological fluctuation in the brain is lacking. We investigated whether familial risk for psychosis (FR) and polygenic risk score for schizophrenia (PRS) are linked with physiological fluctuation in fMRI data. We used data from the Oulu Brain and Mind study ( $N=140-149$, aged 20-24 years) that is a substudy of the Northern Finland Birth Cohort 1986. The participants were scanned with resting-state fMRI. Coefficient of variation (CV) of blood oxygen level dependent (BOLD) signal ( $\left.\mathrm{CV}_{\mathrm{BOLD}}\right)$ was used as a proxy of physiological fluctuation in the brain. Participants who had at least one parent with a previous psychosis were defined as FRs. PRS was computed based on the results of the prior GWAS by the Schizophrenia Working Group. FR or PRS were not associated with $\mathrm{CV}_{\text {BOLD }}$ in cerebrospinal fluid, white matter, or grey matter. The findings did not provide evidence for the previous suggestions that genetic vulnerabilities for schizophrenia become apparent in alterations of the variation of the BOLD signal in the brain.
\end{abstract}

\title{
Keywords
}

Psychosis; Schizophrenia; Polygenic risk score; Familial risk; fMRI; Physiological fluctuation 


\section{Introduction}

The strongest known risk factor predicting onset of schizophrenia and other psychotic disorders is familial risk (Mäki et al. 2005). Roughly $10 \%$ of individuals with a first-degree relative with schizophrenia develop the disorder (Gottesman et al. 2010). The heritability estimate of schizophrenia is approximately 80-90\% (Rijsdijk et al., 2011; Sullivan et al., 2003). Recently, polygenic risk score for schizophrenia (PRS) has been introduced as a new method to estimate genetic risk for developing schizophrenia or other psychotic disorder (Vassos et al. 2016). Previously, it has been highlighted that the association of schizophrenia-related genes with symptom onset may be mediated via genetically determined alterations in the cerebral physiological processes (Birnbaum \& Weinberger, 2013). That is, schizophrenia-related genes may predispose to neurodevelopmental impairments in the formation of brain networks that, in turn, increase the risk for the onset of psychotic symptoms. (Birnbaum \& Weinberger, 2013).

A variety of studies have established that there exist structural and functional brain abnormalities in individuals with familial risk for schizophrenia. Meta-analyses of fMRI studies have shown that familial risk for psychosis is linked to altered neuronal activity particularly in the right temporal and frontal lobes, left thalamus and left cerebellum, and striatum (Cooper et al., 2014; Fusar-Poli et al., 2007). Additionally, there is evidence that polygenic risk score for schizophrenia is related to neural inefficiency in the left dorsolateral prefrontal cortex (Walton et al., 2013). In our previous study, we found that high PRS (vs. low PRS) relates to lower interregional correlations of BOLD fMRI signal and grey-matter volume within the face-processing network (Lieslehto et al., 2018).

Usually, functional magnetic resonance imaging (fMRI) studies aim to examine neuronal-activation-induced changes in the blood-oxygenation-level-dependent (BOLD) signal (Biswal et al., 1996). Importantly, however, the BOLD signal is affected also by a variety of other 
activities than those of neuronal origin, for example, cerebral blood volume and flow and oxygen and carbon dioxide extraction (Cheng et al., 2015; Krüger \& Glover, 2001; Murphy et al., 2009; Wise et al., 2004). This physiological fluctuation in BOLD signal, however, is traditionally regarded as noise variation and is excluded from the fMRI data (e.g. Birn et al., 2012). However, physiological fluctuation provides an important viewpoint to essential neurophysiological processes in the brain. Specifically, cardiac and respiratory cycles induce pulsations in the brain tissues (Kiviniemi et al., 2016; Raitamaa et al., 2018). Moreover, cardiorespiratory frequencies influence the flow of cerebrospinal fluid into the ventricles and conduits (e.g. foramina, subarachnoid space, interstitial space) (Birn et al., 2012; Dreha-Kulaczewski et al., 2015; Kiviniemi et al., 2016). Consequently, it is possible to explore physiological fluctuation in the white matter and cerebrospinal fluid, besides of grey matter (Birn et al., 2006, 2012; Birnbaum \& Weinberger, 2013). Excluding physiological fluctuation from the fMRI data may be a particularly severe limitation in the context of schizophrenia research. It is possible that genetic vulnerabilities for schizophrenia become apparent in specific alterations of the signal-to-noise ratio of the BOLD signal (Harrison \& Weinberger, 2005) that refers to the amount of physiological fluctuation in the brain. Previously, it has been suggested that physiological fluctuation in the white matter is linked with schizophrenia (Cheng et al., 2015) and prodromal symptoms of psychosis (Saarinen et al., submitted). Still, however, no study has investigated whether genetic risk for schizophrenia might be linked with physiological fluctuation in fMRI data.

The aim of the present study was to investigate whether familial risk for psychosis and polygenic risk score for schizophrenia might be linked with physiological fluctuation in the brain. We used data from the Oulu Brain and Mind study (participants were aged 20-24 years) that is a substudy of the Northern Finland Birth Cohort 1986. The participants were scanned with restingstate functional magnetic resonance imaging (r-fMRI). Coefficient of variation (CV) of BOLDsignal $\left(\mathrm{CV}_{\mathrm{BOLD}}\right)$ was used as an indicator of physiological fluctuation in the brain. 


\section{Methods}

\subsection{Participants}

We used data from the Oulu Brain and Mind Study that is a substudy of the Northern Finland Birth Cohort 1986 (NFBC 1986) study. The NFBC 1986 includes individuals with an expected date of birth between July 1985 and June 1986 in the two northernmost provinces of Finland. The original sample of the NFBC 1986 consisted of 9432 participants. The data collection began prospectively before the birth of the participants and has continued since then. For further information of the NFBC 1986, see Järvelin et al. (1997).

The Oulu Brain and Mind Study, a field study with brain-imaging, was conducted in 2007-2010 for a subsample of the NFBC 1986. The primary aim of the Oulu Brain and Mind study was to examine the early neurophysiological alterations in the brain functioning among young individuals with high risk for psychosis. The sample (total $N=329$ ) consisted of 5 groups: (i) participants with familial risk for psychosis, (ii) participants with symptomatic risk for psychosis, (iii) participants with previous psychosis, (iv) participants with attention-deficit/hyperactivity disorder, and (v) healthy controls. When selecting the participants for the Oulu Brain and Mind study, data from health care registers and previous follow-ups of the NFBC 1986 were used. More information about the Oulu Brain and Mind Study is available elsewhere (Veijola et al., 2013).

The design of the NFBC 1986 study and the Oulu Brain and Mind Study were approved by the Ethics Committee of the Northern Ostrobothnia Hospital District in Finland. Moreover, the studies were carried out in accordance with the Declaration of Helsinki. All the participants provided written informed consent after the nature of the procedures was fully explained. 
In this study, we first excluded all the participants with a positive urine drug test for opiates, benzodiazepines, and cannabis $(N=22)$; participants with current use of benzodiazepines, neuroleptics, or other non-specified psychiatric medication $(N=17)$; inadequate or missing brain scan data $(N=12)$; or missing data about educational level $(N=1)$. This resulted in a sample of 277 participants. Thereafter, we included in the analyses all the participants with full data on study variables (familial risk for schizophrenia or PRS; control variables, i.e. age, sex, educational level, and absolute and relative displacement). Consequently, the final sample consisted of 140-149 participants in the analyses.

\subsection{Measures}

\subsubsection{Familial risk for psychosis}

Participants with familial risk for psychosis consisted of the NFBC 1986 participants who had at least one parent with a non-organic psychosis or cluster A personality disorder (paranoid, schizotypal, and schizoid) on the basis of the Finnish Hospital Discharge Register (FHDR) between 1972-2005 were invited to this study as participants with familial risk for psychosis. Cluster A personality disorders were included because of the genetic closeness to schizophrenic psychosis (Tienari et al., 2003).

The FHDR covers all the Finnish mental and general hospitals, beds in local health centres, and private hospitals. During that time ICD-8, ICD-9 and ICD-10 were used, and respective ICD codes either from principal or subsidiary diagnoses were collected. The ICD diagnoses used for psychotic disorders in ICD-8 and ICD-9 were codes 295-299, 3010, 3012 and in ICD-10: codes F20-33 (except non-psychotic mood disorders), F600 and F601. In the NFBC 1986 sample, altogether 272 participants with familial risk were detected. Of them, 1 participant died, 5 
participants were living abroad, and 4 participants could not be reached. Consequently, a total of 262 individuals with familial risk were sent an invitation letter.

\subsubsection{Polygenic risk score for schizophrenia (PRS)}

Detailed information on the collection of the genetic samples and quality control of the genomewide data are provided in a previous study (Lieslehto et al., 2018). Briefly, the results of the genome wide association studies (GWAS) by the Schizophrenia Working Group of the Psychiatric Genomics Consortium (Ripke et al., 2014) were used for the calculation of PRS for schizophrenia in the NFBC 1986 sample. We calculated the score on single-nucleotide polymorphisms (SNPs) reaching genome-wide significance $\left(p=5 \times 10^{-8}\right)$. A total of 112 SNPs were found in the imputed NFBC 1986 GWAS dataset. For each participant, we calculated the sum of the risk SNPs multiplied by the effect size (logarithm of the odds ratio for schizophrenia) as has been done previously (Kendler, 2016). PRS was adjusted for four principal components to account for population stratification since the presence of a systematic difference in allele frequencies between subpopulations of a population may lead to both type I and II errors (Price et al., 2010).

\subsubsection{Participants' background characteristics}

Background information was collected about participants' age, sex, educational level, full-scale intelligence quotient, smoking status, alcohol use, current Axis-I disorders, psychotic-like symptoms, presence of neurological disorders, times of having been unconscious, and level of functioning.

Educational level was assessed with a self-report questionnaire. Educational level was classified 1 (comprehensive school or less) or 2 (matriculation). Full-scale intelligence quotient (FSIQ) was assessed using the Vocabulary and Matrix Reasoning Scales of the Wechsler Adult Intelligence Scale III (WAIS-III, Finnish Edition) (Wechsler et al., 1997). The validity and 
reliability of the WAIS-III are demonstrated to be excellent (e.g. Ryan \& Ward, 1999). As in previous research (e.g. Jukuri et al., 2013), full-scale intelligence quotient was defined as the mean of the two scales.

Smoking status was assessed by asking participants whether they had smoked cigarettes regularly during their life (1=no; $2=$ yes). With regard to alcohol use, participants rated the statement of "I drink too much alcohol or get drunk" with a 3-point scale $(0=$ not true; $1=$ somewhat or sometimes true; $2=$ very true or often true). Participants rating "very true or often true" were defined to have risky alcohol use.

Current Axis-I disorders were assessed with Structured Interview for DSM-IV Axis I Disorders (SCID-I) (First et al., 2002). The presence of neurological disorders and times of having been unconscious were evaluated in the interview of neurological symptoms.

Psychotic-like symptoms were assessed with the Structured Interview for Prodromal Syndromes (SIPS, version 3.0) (Miller et al., 2003). The SIPS measures three separate prodromal syndromes: brief intermittent psychotic syndrome, attenuated positive prodromal syndrome, and genetic risk and deterioration syndrome. Each participants' psychotic symptomatology was defined as the highest score of the SIPS positive symptoms within the past month, ranging from 0 (absence of psychotic symptoms) to 6 (psychosis). This score was further recoded into 4 categories: (i) the score of 0 (no psychotic symptoms); (ii) the scores of 1-2 (mild symptoms); (iii) the scores of 3-5 (prodromal symptoms of psychosis); (iv) the score of 6 (psychotic symptoms).

Level of functioning was evaluated with the Global Assessment of Functioning Scale (GAF) (Spitzer et al., 1996). The psychometric properties of the GAF are shown to be adequate (Sonesson et al., 2010; Startup et al., 2002). The values of the GAF range from 0 to 100, with a higher score of the GAF referring to a higher level of social, occupational, and psychological level of functioning. 


\subsection{Brain-imaging methods and data pre-processing}

Resting-state BOLD (Blood Oxygen Level Dependent-signal) data were collected on a General Electric Signa 1.5 T whole body system with an eight channel receive coil, using an EPI (Echo Planar Imaging) GRE (Gradient Echo) sequence TR (Repetition Time) 1800 ms, TE (Echo Time) $40 \mathrm{~ms}, 280$ time points, 28 oblique axial slices, slice thickness $4 \mathrm{~mm}$, inter-slice space $0.4 \mathrm{~mm}$, covering the whole brain, FOV (Field of View) $25.6 \mathrm{~cm} \times 25.6 \mathrm{~cm}$, with $64 \times 64$ matrix, parallel imaging factor 2 , and a flip angle of $90^{\circ}$. T1-weighted scans were imaged using a 3D FSPGR (Fast Spoiled Gradient echo) BRAVO (Brain Volume imaging) sequence (TR $12.1 \mathrm{~ms}$, TE $5.2 \mathrm{~ms}$, slice thickness $1.0 \mathrm{~mm}$, FOV $24.0 \mathrm{~cm}$, matrix $256 \times 256$, and flip angle $20^{\circ}$ ), and NEX (Number of Excitations) 1 in order to obtain anatomical images for co-registration of the fMRI data to standard space coordinates. For further information, see Jukuri et al. (2013).

Neuroimaging data were analysed with FSL (http://www.fmrib.ox.ac.uk/fsl, FSL

5.0.8) [31-36] and AFNI [37]. We performed the following steps in preprocessing: brain extraction with AFNI's 3dSkullstrip (Cox, 1996; Smith, 2002), motion correction with MCFLIRT [Jenkinson et al., 2002], spatial smoothing with a Gaussian kernel of FWHM $5.0 \mathrm{~mm}$, co-registration with FLIRT, non-linear normalization of structural data to $2 \mathrm{~mm}$ MNI-152 template using FNIRT (Jenkinson \& Smith, 2001; Jenkinson et al., 2002), and detrending with AFNI's 3dDetrend due to the potential scanner-related effect on standard deviation. Relative and absolute root-meansquare (RMS) head displacement (millimeter) was determined from FSL's MCFLIRT and used as covariates in the model. Average absolute displacement refers to the mean of head motion in fMRI time-series in relation to a reference time point (i.e. the first volume in time-series). Average relative displacement, in turn, represents the mean head motion in fMRI time-series in relation to the subsequent time point. FSL's FAST was used for the segmentation of T1-weighted structural images into white matter (WM), grey matter (GM) and cerebrospinal fluid (CSF). 
For each study participant, $\mathrm{CV}_{\mathrm{BOLD}}$ map was calculated as a ratio between standard deviation of preprocessed BOLD-time series divided by the mean of preprocessed BOLD-time series in each voxel. This method has been used by (Kananen et al., 2018; Jahanian et al., 2017; Tuovinen et al., 2017, 2018) and is similar to the method used by (Makedonov et al., 2013, 2016). A close concept for $\mathrm{CV}_{\mathrm{BOLD}}$ is signal-to-noise ratio that is calculated as $1 / \mathrm{CV}_{\mathrm{BOLD}}$ and has been used previously (e.g. Harrison \& Weinberger, 2005). Next, we extracted the average $\mathrm{CV}_{\text {BOLD }}$ in WM, GM and CSF (segmented with FSL's FAST). Sex, age, education, absolute and relative displacement were used as covariates in the model.

\subsection{Statistical analyses}

The statistical analyses were run using STATA SE (version 15.0). The associations of familial risk for psychosis and polygenic risk score for schizophrenia with $\mathrm{CV}_{\mathrm{BOLD}}$ in the brain were investigated using linear regression analyses. Familial risk for psychosis and polygenic risk score for schizophrenia were added separately to the model. Further, separate models were estimated for $\mathrm{CV}_{\mathrm{BOLD}}$ in CSF, WM, and GM. As additional analyses, all the analyses were run among those participants with no psychotic-like symptomatology (the current SIPS score less than 3). All the analyses were adjusted for age, sex, educational level, absolute and relative displacement. We assessed the level of multicollinearity using the value of VIF (variance inflation factor). VIF values less than 10 are stated to indicate low level of multicollinearity (see Alin, 2010). In our analyses, the mean VIF was 1.09 (VIF varied between 1.01-1.20), indicating that there was not significant multicollinearity.

\section{Results}


Descriptive statistics of the study variables are shown in Table 1. In the sample, there were 72 participants (34.3\%) with familial risk for psychosis.

[Table 1 about here]

Table 2 shows the results of the regression analyses, when predicting CV $\mathrm{BOLD}_{\text {in }}$ CSF, WM, and GM by familial risk for psychosis. Familial risk for psychosis was not related to $\mathrm{CV}_{\text {BOLD }}$ in CSF, $\mathrm{WM}$, or GM. Table 3 presents the results of the regression analyses, when predicting $\mathrm{CV}_{\mathrm{BOLD}}$ in CSF, WM, and GM by polygenic risk score for schizophrenia. Polygenic risk score for schizophrenia was not associated with $\mathrm{CV}_{\mathrm{BOLD}}$ in CSF, WM, or GM. All the findings were adjusted for age, gender, educational level, and absolute and relative displacement.

As additional analyses, the regression analyses were rerun among participants without psychotic-like symptoms (the current SIPS $<3, N=132$ for familial risk analyses, $N=133$ for PRS analyses). All the associations remained non-significant. The results of the additional analyses are described with more detail in Supplementary Tables 1-2.

Finally, we investigated the associations of familial risk for psychosis and polygenic risk score for schizophrenia with parameters of motion in the fMRI data. Familial risk for psychosis was not linked with absolute (beta $=-0.093, p=0.283$ ) or relative parameters of motion (beta=0.100, $p=0.246$ ). Moreover, polygenic risk score was not linked with absolute (beta $=-0.064, p=0.439$ ) or relative parameters of motion (beta $=-0.074, p=0.375$ ). For further information, see Supplementary Tables 3-4.

[Table 2 about here]

[Table 3 about here] 


\section{Discussion}

To the best of our knowledge, this study was the first to investigate whether familial risk for psychosis and polygenic risk score for schizophrenia might be related to physiological fluctuation (variation of the BOLD signal) in the brain. The results showed that neither familial risk for psychosis nor polygenic risk score was associated with lower $\mathrm{CV}_{\mathrm{BOLD}}$ in cerebrospinal fluid, white matter, or grey matter. All the findings were controlled for age, sex, educational level, and absolute and relative displacement. Finally, all the results remained non-significant when including only those participants with no psychotic-like symptoms (the current SIPS score < 3). Taken together, familial or genetic risk for psychosis appeared not to be related to physiological fluctuation (variation of the BOLD signal) in the brain.

Previous studies have shown that physiological fluctuation is related to Alzheimer's disease (Makedonov et al., 2016), acute ischemia (Khalil et al., 2017), epilepsy (Kananen et al., 2018), schizophrenia (Cheng et al., 2015), and prodromal syndromes of psychosis (Saarinen et al., submitted). This study was the first to investigate whether physiological fluctuation in the fMRI data is linked with genetic factors. Previously, it has been postulated that genetic vulnerabilities for schizophrenia become apparent in specific alterations of the signal-to-noise ratio (Harrison \& Weinberger, 2005) that refers to the variance of BOLD signal in the brain. However, the present study did not provide evidence for this postulation. It seems that the changes in the physiological fluctuation of the brain are evident only after the onset of neuropsychiatric diseases.

The null results about the link between psychosis-related genes and physiological fluctuation in the brain may have several explanations. Firstly, there is previous evidence for the heritability of heart rate variability (Kupper et al., 2004; Snieder et al., 2007), ambulatory cardiorespiratory coupling (Kupper et al., 2005), systemic arterial stiffness (Snieder et al., 2000), and respiratory control (Gaultier, 2004). These qualities of cardiorespiratory functioning, in turn, 
are closely related to the amount of physiological fluctuation in the brain (e.g. Birn et al., 2012; Cheng et al., 2015; Krüger \& Glover, 2001; Murphy et al., 2009; Wise et al., 2004). The current study indicates that the genes, which are related to the elements of physiological fluctuation, may not be overlapping with the schizophrenia-related genes. Secondly, it may be that schizophreniarelated genes are linked to physiological fluctuation in the brain only when co-occurring with some other genes. For example, one study reported that dopamine-related genes are related to cortical signal-to-noise ratio but only in the presence of another genetic polymorphism (in the gene for the enzyme catechol-o-methyltransferase) (Winterer \& Weinberger, 2003).

Thirdly, it might be speculated that our null results might be accounted by a compensatory effect. That is, for example, individuals with prodromal symptoms have increased activity in certain brain regions during prodromal symptoms, but decreased activity during improvement of the symptoms (Fusar-Poli et al., 2010). Thus, individuals at risk for psychosis may need more neurophysiological effort in order to achieve the same level of brain functioning (Cooper et al., 2014). Similarly, some degree of physiological fluctuation (signal-to-noise ratio) appears to be necessary for adequate brain functioning (e.g. Birn et al., 2012; Dreha-Kulaczewski et al., 2015; Kiviniemi et al., 2016). This is because signal-to-noise ratio partly reflects the rhythm of arterial pulsations that, in turn, influences the flow of cerebrospinal fluid into perivascular spaces and induce clearance of metabolic waste (Mestre et al., 2018). Accordingly, individuals at genetic risk for psychosis might have an innate susceptibility for increased physiological fluctuation in the brain, but they might have compensated it and, in this way, captured the "normal" level of fluctuation. This might possibly explain why we did not obtain any associations between schizophrenia-related genes and alterations in the level of physiological fluctuation.

This study has some limitations that are necessary to be taken into consideration. Firstly, our samples were relatively small in the analyses $(N=140-149)$. Previously, it has been stated that in the context of complex genetic disorders, a small sample size may result in the lack of 
power to obtain any associations between genetic factors and disease-related neurophysiological alterations (Harrison \& Weinberger, 2005). In this study, all the associations of familial risk and polygenic risk score with $\mathrm{CV}_{\mathrm{BOLD}}$ in the cerebrospinal fluid, white matter, and grey matter were statistically non-significant. However, the null results may not likely be accounted by the relatively small sample size because gender, for example, was significantly associated with $\mathrm{CV}_{\mathrm{BOLD}}$ in the brain in our sample.

Secondly, our sample was relatively heterogeneous, including participants with familial risk for psychosis, earlier adolescent attention-deficit hyperactivity disorder, or current Axis-I disorder. There is previous evidence that psychotic symptoms have a high comorbidity level with other psychiatric and neurological symptoms, for example, depression, anxiety, epilepsy, ADHD, and substance use disorders (Fusar-Poli et al., 2012; Gaitatzis et al., 2004; Karatekin et al., 2010; Keshavan et al., 2003; Schuckit et al., 2006). Hence, excluding all the individuals with any other psychiatric or neurological diagnosis might result in a substantial selective bias in the sample and limit the generalizability of the findings to other populations. Another possibility might have been to control for all the other psychiatric and neurological diagnoses. This method, however, would likely have resulted in a large number of covariates and statistical problems with multicollinearity. Consequently, we ended up to control for the basic covariates in the analyses (i.e. sex, age, educational level, and absolute and relative displacement).

The current study had also a variety of strengths. Overall, this study was the first to investigate whether genetic vulnerabilities for psychosis might be linked with $\mathrm{CV}_{\mathrm{BOLD}}$ in the brain. Additionally, we had two different indicators of genetic vulnerabilities: familial risk for psychosis and polygenic risk score for schizophrenia. This is line with a previous statement that when investigating neuroimaging-based phenotypes for schizophrenia, it is necessary to obtain the findings both among individuals with a high genetic risk score and among first-degree relatives of psychotic individuals (Birnbaum \& Weinberger, 2013). Secondly, we used whole-brain indicators 
of $\mathrm{CV}_{\mathrm{BOLD}}$ in grey matter, white matter, and cerebrospinal fluid, instead of investigating selectively some brain regions of interest. Thirdly, familial risk for psychosis was assessed reliably on the basis of the Finnish Hospital Discharge Register. The PRS, in turn, was based on a total of 112 SNPs derived from genome wide association studies. Fourthly, the NFBC 1986 study provided a sample of roughly same-aged participants in their young adulthood. This is in line with previous recommendations that the neurophysiological correlates of schizophrenia should be investigated in the early adulthood in order to ensure that the findings are present prior to the onset of illness (Cooper et al., 2014).

In conclusion, this study showed that genetic vulnerabilities for schizophrenia were not related to physiological fluctuation (amount of variation of the BOLD signal) in the brain. With regard to future studies, the measures of $\mathrm{CV}_{\mathrm{BOLD}}$ are close to the recently detected BOLD delay maps in acute ischemia and small vessel disease (see Khalil et al., 2017; Lv et al., 2013; Tong et al., 2017). Hence, the link of psychosis with the BOLD delay maps could be addressed in the future.

\section{References}

Alin, A., 2010. Multicollinearity. Wiley Interdisciplinary Reviews: Computational Statistics, 2, 370-374. https://doi.org/10.1002/wics.84

Birn, R.M., 2012. The role of physiological noise in resting-state functional connectivity. Neuroimage 62, 864-870. https://doi.org/10.1016/j.neuroimage.2012.01.016

Birn, R.M., Diamond, J.B., Smith, M.A., Bandettini, P.A., 2006. Separating respiratory-variationrelated fluctuations from neuronal-activity-related fluctuations in fMRI. Neuroimage 31, 1536-1548. https://doi.org/10.1016/j.neuroimage.2006.02.048

Birnbaum, R., Weinberger, D.R., 2013. Functional neuroimaging and schizophrenia: a view towards effective connectivity modeling and polygenic risk. Dialogues in Clinical Neuroscience 15, 279-289. 
Biswal, B., Deyoe, E. A., Hyde, J. S., 1996. Reduction of physiological fluctuations in fMRI using digital filters. Magnetic Resonance in Medicine 35, 107-113. https://doi.org/10.1002/mrm.1910350114

Cheng, H., Newman, S.D., Kent, J.S., Bolbecker, A., Klaunig, M.J., O’Donnell, B.F., ... Hetrick, W.P., 2015. White matter abnormalities of microstructure and physiological noise in schizophrenia. Brain Imaging and Behavior 9, 868-877. https://doi.org/10.1007/s11682-0149349-1

Cooper, D., Barker, V., Radua, J., Fusar-Poli, P., Lawrie, S.M., 2014. Multimodal voxel-based meta-analysis of structural and functional magnetic resonance imaging studies in those at elevated genetic risk of developing schizophrenia. Psychiatry Research: Neuroimaging 221, 69-77. https://doi.org/10.1016/j.pscychresns.2013.07.008

Dreha-Kulaczewski, S., Joseph, A. A., Merboldt, K. D., Ludwig, H. C., Gärtner, J., Frahm, J., 2015. Inspiration is the major regulator of human CSF flow. Journal of Neuroscience 35, 2485-2491. https://doi.org/10.1523/JNEUROSCI.3246-14.2015

First, M.B., Spitzer, R.L., Gibbon, M., Williams, J.B., 2002. Structured Clinical Interview for DSM-IV-TR Axis I Disorders, Research Version, Patient Edition. SCID-I/P.

Fusar-Poli, P., Broome, M.R., Matthiasson, P., Woolley, J.B., Johns, L.C., Tabraham, P., ...McGuire, P., 2010. Spatial working memory in individuals at high risk for psychosis: longitudinal fMRI study. Schizophrenia Research 123, 45-52. https://doi.org/10.1016/j.schres.2010.06.008

Fusar-Poli, P., Nelson, B., Valmaggia, L., Yung, A.R., McGuire, P.K., 2012. Comorbid depressive and anxiety disorders in 509 individuals with an at-risk mental state: impact on psychopathology and transition to psychosis. Schizophrenia Bulletin 40, 120-131. https://doi.org/10.1093/schbul/sbs136 
Fusar-Poli, P., Perez, J., Broome, M., Borgwardt, S., Placentino, A., Caverzasi, E., ... McGuire, P., 2007. Neurofunctional correlates of vulnerability to psychosis: a systematic review and metaanalysis. Neuroscience \& Biobehavioral Reviews 31, 465-484.

https://doi.org/10.1016/j.neubiorev.2006.11.006

Gaitatzis, A., Trimble, M.R., Sander, J.W., 2004. The psychiatric comorbidity of epilepsy. Acta Neurologica Scandinavica 110, 207-220. https://doi.org/10.1111/j.1600-0404.2004.00324.X

Gaultier, C., 2004. Genes and genetics in respiratory control. Paediatric Respiratory Reviews 5, 166-172. https://doi.org/10.1016/j.prrv.2004.04.012

Gottesman, I.I., Laursen, T.M., Bertelsen, A., Mortensen, P.B., 2010. Severe mental disorders in offspring with 2 psychiatrically ill parents. Archives of General Psychiatry 67, 252-257. https://doi.org/10.1001/archgenpsychiatry.2010.1

Harrison, P.J., Weinberger, D.R., 2005. Schizophrenia genes, gene expression, and neuropathology: on the matter of their convergence. Molecular Psychiatry 10, 40. https://doi.org/10.1038/sj.mp.4001558

Jahanian, H., Christen, T., Moseley, M. E., Pajewski, N. M., Wright, C. B., Tamura, M. K., ... SPRINT Study Research Group, 2017. Measuring vascular reactivity with resting-state blood oxygenation level-dependent (BOLD) signal fluctuations: A potential alternative to the breathholding challenge. Journal of Cerebral Blood Flow \& Metabolism 37, 2526-2538. https://doi.org/10.1177/0271678X16670921

Jukuri, T., Kiviniemi, V., Nikkinen, J., Miettunen, J., Mäki, P., Jääskeläinen, E., Mukkala, S., Koivukangas, J., Nordström, T., Taanila, A., Moilanen, I., Heinimaa, M., Barnett, J.H., Jones, P. B., Murray, G.K., Moilanen, I., 2013. Default mode network in young people with familial risk for psychosis - the Oulu Brain and Mind study. Schizophrenia Research 143, 239-245. https://doi.org/10.1016/j.schres.2012.11.020 
Järvelin, M.R., Elliott, P., Kleinschmidt, I., Martuzzi, M., Grundy, C., Hartikainen, A.L., Rantakallio, P., 1997. Ecological and individual predictors of birthweight in a northern Finland birth cohort 1986. Paediatric and Perinatal Epidemiology 11, 298-312. https://doi.org/10.1111/j.1365-3016.1997.tb00007.x

Karatekin, C., White, T., Bingham, C., 2010. Shared and nonshared symptoms in youth-onset psychosis and ADHD. Journal of Attention Disorders 14,121-131. https://doi.org/10.1177/1087054709347434

Kendler, K.S., 2016. The schizophrenia polygenic risk score: to what does it predispose in adolescence? JAMA Psychiatry 73, 193-194. https://doi.org/10.1001/jamapsychiatry.2015.2964

Keshavan, M.S., Sujata, M., Mehra, A., Montrose, D.M., Sweeney, J.A., 2003. Psychosis proneness and ADHD in young relatives of schizophrenia patients. Schizophrenia Research 59, 85-92. https://doi.org/10.1016/S0920-9964(01)00400-5

Khalil, A.A., Ostwaldt, A.C., Nierhaus, T., Ganeshan, R., Audebert, H.J., Villringer, K., ... Fiebach, J.B., 2017. Relationship between changes in the temporal dynamics of the blood-oxygen-leveldependent signal and hypoperfusion in acute ischemic stroke. Stroke 48, 925-931. https://doi.org/10.1161/STROKEAHA.116.015566

Krüger, G., Glover, G.H., 2001. Physiological noise in oxygenation-sensitive magnetic resonance imaging. Magnetic Resonance in Medicine: An Official Journal of the International Society for Magnetic Resonance in Medicine 46, 631-637. https://doi.org/10.1002/mrm.1240

Kupper, N.H., Willemsen, G., van den Berg, M., de Boer, D., Posthuma, D., Boomsma, D.I., de Geus, E.J., 2004. Heritability of ambulatory heart rate variability. Circulation 110, 2792-2796. https://doi.org/10.1161/01.CIR.0000146334.96820.6E

Kupper, N., Willemsen, G., Posthuma, D., De Boer, D., Boomsma, D.I., De Geus, E.J., 2005. A genetic analysis of ambulatory cardiorespiratory coupling. Psychophysiology 42, 202-212. https://doi.org/10.1111/j.1469-8986.2005.00276.x 
Lieslehto, J., Kiviniemi, V.J., Nordström, T., Barnett, J.H., Murray, G.K., Jones, P.B., ... Veijola, J., 2018. Polygenic Risk Score for Schizophrenia and Face-Processing Network in Young Adulthood. Schizophrenia Bulletin. https://doi.org/10.1093/schbul/sby139

Lv, Y., Margulies, D. S., Cameron Craddock, R., Long, X., Winter, B., Gierhake, D., ... Villringer, A., 2013. Identifying the perfusion deficit in acute stroke with resting-state functional magnetic resonance imaging. Annals of Neurology 73, 136-140. https://doi.org/10.1002/ana.23763

Makedonov, I., Black, S.E., MacIntosh, B.J., 2013. BOLD fMRI in the white matter as a marker of aging and small vessel disease. PloS One 8, e67652.

https://doi.org/10.1371/journal.pone.0067652

Makedonov, I., Chen, J.J., Masellis, M., MacIntosh, B.J., Alzheimer's Disease Neuroimaging Initiative, 2016. Physiological fluctuations in white matter are increased in Alzheimer's disease and correlate with neuroimaging and cognitive biomarkers. Neurobiology of Aging 37, 12-18. https://doi.org/10.1016/j.neurobiolaging.2015.09.010

Mestre, H., Tithof, J., Du, T., Song, W., Peng, W., Sweeney, A. M., ... \& Kelley, D. H. (2018). Flow of cerebrospinal fluid is driven by arterial pulsations and is reduced in hypertension. Nature Communications, 9, 4878. https://doi.org/10.1038/s41467-018-07318-3

Miller, T.J., McGlashan, T.H., Rosen, J.L., Cadenhead, K., Ventura, J., McFarlane, W., Perkins, D. O., Pearlson, G.D., Woods, S.W., 2003. Prodromal assessment with the structured interview for prodromal syndromes and the scale of prodromal symptoms: predictive validity, interrater reliability, and training to reliability. Schizophrenia Bulletin 29, 703-715. https://doi.org/10.1093/oxfordjournals.schbul.a007040

Murphy, K., Birn, R.M., Handwerker, D.A., Jones, T.B., Bandettini, P.A., 2009. The impact of global signal regression on resting state correlations: are anti-correlated networks introduced. Neuroimage 44, 893-905. https://doi.org/10.1016/j.neuroimage.2008.09.036 
Mäki, P., Veijola, J., Jones, P.B., Murray, G.K., Koponen, H., Tienari, P., ... Lauronen, E., 2005. Predictors of schizophrenia—a review. British Medical Bulletin 73, 1-15. https://doi.org/10.1093/bmb/ldh046

Price, A. L., Zaitlen, N. A., Reich, D., Patterson, N., 2010. New approaches to population stratification in genome-wide association studies. Nature Reviews Genetics 11, 459-463. https://doi.org/10.1038/nrg2813

Raitamaa, L., Korhonen, V., Huotari, N., Raatikainen, V., Hautaniemi, T., Kananen, J., ... Borchardt, V., 2018. Breath hold effect on cardiovascular brain pulsations-A multimodal magnetic resonance encephalography study. Journal of Cerebral Blood Flow \& Metabolism, 0271678X18798441. https://doi.org/10.1177/0271678X18798441

Rijsdijk, F.V., Gottesman, I.I., McGuffin, P., Cardno, A.G., 2011. Heritability estimates for psychotic symptom dimensions in twins with psychotic disorders. American Journal of Medical Genetics Part B: Neuropsychiatric Genetics 156, 89-98. https://doi.org/10.1002/ajmg.b.31145

Ripke, S., Neale, B.M., Corvin, A., Walters, J.T., Farh, K.H., Holmans, P.A., ... Pers, T.H., 2014. Biological insights from 108 schizophrenia-associated genetic loci. Nature 511, 421. https://doi.org/10.1038/nature13595

Ryan, J.J., Ward, L.C., 1999. Validity, reliability, and standard errors of measurement for two seven-subtest short forms of the Wechsler Adult Intelligence Scale-III. Psychological Assessment 11, 207-211.

Schuckit, M.A., 2006. Comorbidity between substance use disorders and psychiatric conditions. Addiction 101, 76-88. https://doi.org/10.1111/j.1360-0443.2006.01592.x

Snieder, H., Hayward, C.S., Perks, U., Kelly, R.P., Kelly, P.J., Spector, T.D., 2000. Heritability of central systolic pressure augmentation: a twin study. Hypertension 35, 574-579. 
Snieder, H., Van Doornen, L.J., Boomsma, D.I., Thayer, J.F., 2007. Sex differences and heritability of two indices of heart rate dynamics: a twin study. Twin Research and Human Genetics 10, 364-372. https://doi.org/10.1375/twin.10.2.364

Sonesson, O., Tjus, T., Arvidsson, H., 2010. Reliability of a functioning scale (GAF) among psychiatric ward staff. Nordic Psychology 62, 53-64. https://doi.org/10.1027/1901$\underline{2276 / a 000005}$

Spitzer, R.L., Gibbon, M., Williams, J.B.W., Endicott, J., 1996. Global assessment of functioning (GAF) scale. Outcome Assessment in Clinical Practice, 76-78.

Startup, M., Jackson, M.C., Bendix, S., 2002. The concurrent validity of the Global Assessment of Functioning (GAF). British Journal of Clinical Psychology 41, 417-422. https://doi.org/10.1348/014466502760387533

Sullivan, P.F., Kendler, K.S., Neale, M.C., 2003. Schizophrenia as a complex trait: evidence from a meta-analysis of twin studies. Archives of General Psychiatry 60, 1187-1192. https://doi.org/10.1001/archpsyc.60.12.1187

Tienari, P., Wynne, L. C., Läksy, K., Moring, J., Nieminen, P., Sorri, A., ..., Wahlberg, K. E., 2003. Genetic boundaries of the schizophrenia spectrum: evidence from the Finnish Adoptive Family Study of Schizophrenia. American Journal of Psychiatry 160, 1587-1594. https://doi.org/10.1176/appi.ajp.160.9.1587

Tong, Y., Lindsey, K. P., Hocke, L. M., Vitaliano, G., Mintzopoulos, D., Frederick, B. D., 2017. Perfusion information extracted from resting state functional magnetic resonance imaging. Journal of Cerebral Blood Flow \& Metabolism 37, 564-576. https://doi.org/10.1177/0271678X16631755

Tuovinen, T., Kananen, J., Rytty, R., Moilanen, V., Elseoud, A.A., Remes, A.M., ... Alzheimer's Disease Neuroimaging Initiative, 2018. Altered BOLD signal variation in Alzheimer's disease and frontotemporal dementia. BioRxiv, 455683. https://doi.org/10.1101/455683 
Tuovinen, T., Rytty, R., Moilanen, V., Abou Elseoud, A., Veijola, J., Remes, A.M., Kiviniemi, V. J., 2017. The effect of gray matter ICA and coefficient of variation mapping of BOLD data on the detection of functional connectivity changes in Alzheimer's disease and bvFTD. Frontiers in Human Neuroscience, 10. https://doi.org/10.3389/fnhum.2016.00680

Vassos, E., Di Forti, M., Coleman, J., Iyegbe, C., Prata, D., Euesden, J., ... Newhouse, S., 2017. An examination of polygenic score risk prediction in individuals with first-episode psychosis. Biological Psychiatry 81, 470-477. https://doi.org/10.1016/j.biopsych.2016.06.028 Veijola, J., Mäki, P., Jääskeläinen, E., Koivukangas, J., Moilanen, I., Taanila, A., ... Heinimaa, M., 2013. Young people at risk for psychosis: case finding and sample characteristics of the Oulu Brain and Mind Study. Early Intervention in Psychiatry 7, 146-154. https://doi.org/10.1111/j.1751-7893.2012.00360.x

Walton, E., Geisler, D., Lee, P.H., Hass, J., Turner, J.A., Liu, J., ... Gollub, R.L., 2013. Prefrontal inefficiency is associated with polygenic risk for schizophrenia. Schizophrenia Bulletin 40, 1263-1271. https://doi.org/10.1093/schbul/sbt174

Wechsler, D., Coalson, D.L., Raiford, S.E., 1997. WAIS-III: Wechsler Adult Intelligence Scale. San Antonio, TX: Psychological Corporation.

Winterer, G., Weinberger, D. R., 2003. Cortical signal-to-noise ratio: Insight into the pathophysiology and genetics of schizophrenia. Clinical Neuroscience Research 3, 55-66. https://doi.org/10.1016/S1566-2772(03)00019-7

Wise, R.G., Ide, K., Poulin, M.J., Tracey, I., 2004. Resting fluctuations in arterial carbon dioxide induce significant low frequency variations in BOLD signal. Neuroimage 21, 1652-1664. https://doi.org/10.1016/j.neuroimage.2003.11.025 
Table 1. Means, standard deviations (SD), ranges, and frequencies of the study variables.

\begin{tabular}{|c|c|c|c|c|}
\hline & Mean & SD & Range & Frequency $(\%)$ \\
\hline Age & 22.81 & 0.80 & $20.95 ; 24.64$ & \\
\hline Sex (female) & & & & $69(32.9)$ \\
\hline Familial risk for psychosis & & & & $72(34.3)$ \\
\hline Polygenic risk score for schizophrenia & 0.05 & 0.98 & $-2.10 ; 2.50$ & \\
\hline \multicolumn{5}{|l|}{ SIPS positive symptoms } \\
\hline No psychotic symptoms & & & & $111(52.9)$ \\
\hline Mild symptoms & & & & $81(38.6)$ \\
\hline Prodromal symptoms of psychosis & & & & $17(8.1)$ \\
\hline Psychosis & & & & $1(0.5)$ \\
\hline \multicolumn{5}{|l|}{ Educational level } \\
\hline Comprehensive school or less & & & & $81(38.6)$ \\
\hline University / graduate-level & & & & $129(61.4)$ \\
\hline Smoker & & & & $91(43.3)$ \\
\hline Risky alcohol use & & & & $51(24.3)$ \\
\hline Current Axis-I disorder & & & & $37(26.2)$ \\
\hline Neurological disorder & & & & $33(15.7)$ \\
\hline Current level of functioning & 80.97 & 10.65 & $21 ; 96$ & \\
\hline Full-scale intelligence quotient & 97.14 & 24.36 & $25 ; 160$ & \\
\hline
\end{tabular}


Genetic susceptibilities and physiological fluctuation

Table 2. Results of linear regression analyses, when predicting $\mathrm{CV}_{\mathrm{BOLD}}$ in cerebrospinal fluid, white matter, and grey matter by familial risk for psychosis.

\begin{tabular}{|c|c|c|c|c|c|c|c|c|c|}
\hline & \multicolumn{3}{|c|}{$\mathrm{CV}_{\mathrm{BOLD}}$ in cerebrospinal fluid } & \multicolumn{3}{|c|}{$\mathrm{CV}_{\mathrm{BOLD}}$ in white matter } & \multicolumn{3}{|c|}{$\mathrm{CV}_{\mathrm{BOLD}}$ in grey matter } \\
\hline & Beta & $\mathrm{B}$ & $p$ & Beta & $\mathrm{B}$ & $p$ & Beta & $\mathrm{B}$ & $p$ \\
\hline Age & -0.322 & -0.001 & $<0.001$ & -0.229 & 0.000 & 0.002 & -0.172 & -0.000 & 0.014 \\
\hline $\operatorname{Sex}^{1}$ & -0.361 & -0.002 & $<0.001$ & -0.364 & -0.001 & $<0.001$ & -0.271 & -0.001 & $<0.001$ \\
\hline Educational level & -0.013 & 0.000 & 0.853 & -0.105 & 0.000 & 0.180 & -0.032 & 0.000 & 0.662 \\
\hline Absolute displacement & -0.198 & -0.002 & 0.006 & -0.220 & -0.001 & 0.005 & -0.206 & -0.001 & 0.005 \\
\hline Relative displacement & 0.439 & 0.062 & $<0.001$ & 0.321 & 0.023 & $<0.001$ & 0.548 & 0.055 & $<0.001$ \\
\hline Familial risk for psychosis ${ }^{2}$ & -0.135 & -0.001 & 0.050 & -0.061 & 0.000 & 0.419 & -0.096 & 0.000 & 0.177 \\
\hline
\end{tabular}

${ }^{1}$ Male as the reference group. ${ }^{2}$ Participants without familial risk for psychosis as the reference group. $N=140$ 
Genetic susceptibilities and physiological fluctuation

Table 3. Results of linear regression analyses, when predicting $\mathrm{CV}_{\mathrm{BOLD}}$ in cerebrospinal fluid, white matter, and grey matter by polygenic risk score for schizophrenia.

\begin{tabular}{|c|c|c|c|c|c|c|c|c|c|}
\hline & \multicolumn{3}{|c|}{$\mathrm{CV}_{\mathrm{BOLD}}$ in cerebrospinal fluid } & \multicolumn{3}{|c|}{$\mathrm{CV}_{\mathrm{BOLD}}$ in white matter } & \multicolumn{3}{|c|}{$\mathrm{CV}_{\mathrm{BOLD}}$ in grey matter } \\
\hline & Beta & $\mathrm{B}$ & $p$ & Beta & $\mathrm{B}$ & $p$ & Beta & $\mathrm{B}$ & $p$ \\
\hline Age & -0.279 & -0.001 & $<0.001$ & -0.225 & 0.000 & 0.001 & -0.143 & 0.000 & 0.016 \\
\hline $\operatorname{Sex}^{1}$ & -0.337 & -0.002 & $<0.001$ & -0.345 & -0.001 & $<0.00$ & -0.282 & -0.001 & $<0.001$ \\
\hline Educational level & -0.033 & 0.000 & 0.607 & -0.146 & 0.000 & 0.025 & -0.038 & -0.000 & 0.521 \\
\hline Absolute displacement & -0.276 & -0.003 & $<0.001$ & -0.316 & -0.002 & $<0.00$ & -0.287 & -0.002 & $<0.001$ \\
\hline Relative displacement & 0.569 & 0.064 & $<0.001$ & 0.534 & 0.033 & $<0.00$ & 0.703 & 0.059 & $<0.001$ \\
\hline $\begin{array}{l}\text { Polygenic risk score } \\
\text { for schizophrenia }\end{array}$ & -0.041 & 0.000 & 0.518 & -0.019 & 0.000 & 0.771 & -0.032 & 0.000 & 0.583 \\
\hline
\end{tabular}

${ }^{1}$ Male as the reference group. ${ }^{2}$ The reference group. $N=148$ 
Supplementary Table 1. Results of linear regression analyses, when predicting $\mathrm{CV}_{\mathrm{BOLD}}$ in cerebrospinal fluid, white matter, and grey matter by familial risk for psychosis among participants with current SIPS $<3$.

\begin{tabular}{|c|c|c|c|c|c|c|c|c|c|}
\hline & \multicolumn{3}{|c|}{$\mathrm{CV}_{\mathrm{BOLD}}$ in cerebrospinal fluid } & \multicolumn{3}{|c|}{$\mathrm{CV}_{\mathrm{BOLD}}$ in white matter } & \multicolumn{3}{|c|}{$\mathrm{CV}_{\mathrm{BOLD}}$ in grey matter } \\
\hline & Beta & $\mathrm{B}$ & $p$ & Beta & $\mathrm{B}$ & $p$ & Beta & $\mathrm{B}$ & $p$ \\
\hline Age & -0.291 & -0.001 & $<0.001$ & -0.180 & 0.000 & 0.019 & -0.140 & 0.000 & 0.050 \\
\hline $\operatorname{Sex}^{1}$ & -0.382 & -0.002 & $<0.001$ & -0.375 & -0.001 & $<0.001$ & -0.287 & -0.001 & $<0.001$ \\
\hline Educational level & -0.005 & 0.000 & 0.942 & -0.108 & 0.000 & 0.181 & -0.027 & 0.000 & 0.720 \\
\hline Absolute displacement & -0.209 & -0.002 & 0.005 & -0.235 & -0.001 & 0.004 & -0.217 & -0.001 & 0.004 \\
\hline Relative displacement & 0.459 & 0.064 & $<0.001$ & 0.341 & 0.024 & $<0.001$ & 0.566 & 0.057 & $<0.001$ \\
\hline Familial risk for psychosis ${ }^{2}$ & -0.156 & -0.001 & 0.029 & -0.073 & 0.000 & 0.353 & -0.107 & 0.000 & 0.144 \\
\hline
\end{tabular}

${ }^{1}$ Male as the reference group. ${ }^{2}$ Participants without familial risk for psychosis as the reference group. $N=132$ 
Genetic susceptibilities and physiological fluctuation

Supplementary Table 2. Results of linear regression analyses, when predicting $\mathrm{CV}_{\mathrm{BOLD}}$ in cerebrospinal fluid, white matter, and grey matter by polygenic risk score for schizophrenia among participants with current SIPS $<3$.

\begin{tabular}{|c|c|c|c|c|c|c|c|c|c|}
\hline & \multicolumn{3}{|c|}{$\mathrm{CV}_{\mathrm{BOLD}}$ in cerebrospinal fluid } & \multicolumn{3}{|c|}{$\mathrm{CV}_{\mathrm{BOLD}}$ in white matter } & \multicolumn{3}{|c|}{$\mathrm{CV}_{\mathrm{BOLD}}$ in grey matter } \\
\hline & Beta & $\mathrm{B}$ & $p$ & Beta & $\mathrm{B}$ & $p$ & Beta & $\mathrm{B}$ & $p$ \\
\hline Age & -0.291 & -0.001 & $<0.001$ & -0.225 & 0.000 & $<0.001$ & -0.127 & 0.000 & 0.038 \\
\hline $\operatorname{Sex}^{1}$ & -0.338 & -0.002 & $<0.001$ & -0.352 & -0.001 & $<0.001$ & -0.290 & -0.001 & $<0.001$ \\
\hline Educational level & -0.024 & 0.000 & 0.715 & -0.145 & 0.000 & 0.028 & -0.041 & -0.002 & $<0.001$ \\
\hline Absolute displacement & -0.268 & -0.003 & $<0.001$ & -0.311 & -0.002 & $<0.001$ & -0.284 & 0.059 & $<0.001$ \\
\hline Relative displacement & 0.594 & 0.066 & $<0.001$ & 0.572 & 0.034 & $<0.001$ & 0.714 & 0.059 & $<0.001$ \\
\hline $\begin{array}{l}\text { Polygenic risk score for } \\
\text { schizophrenia }\end{array}$ & -0.045 & 0.000 & 0.485 & -0.026 & 0.000 & 0.685 & -0.031 & 0.000 & 0.607 \\
\hline
\end{tabular}

${ }^{1}$ Male as the reference group. ${ }^{2}$ The reference group. $N=133$ 
Supplementary Table 3. Results of linear regression analyses, when predicting absolute and relative motion by familial risk for psychosis.

\begin{tabular}{llllllll}
\hline & \multicolumn{3}{c}{ Absolute motion } & & \multicolumn{3}{c}{ Relative motion } \\
\cline { 2 - 3 } \cline { 6 - 8 } & Beta & B & $p$ & & Beta & B & $p$ \\
\hline Age & -0.067 & -0.031 & 0.439 & & -0.043 & -0.001 & 0.614 \\
Sex & -0.102 & -0.074 & 0.245 & & -0.077 & -0.003 & 0.379 \\
Educational level & 0.042 & 0.031 & 0.639 & & 0.168 & 0.007 & 0.059 \\
Familial risk for psychosis & -0.093 & -0.066 & 0.283 & & 0.100 & 0.004 & 0.246 \\
\hline
\end{tabular}

${ }^{1}$ Male as the reference group. ${ }^{2}$ Participants without familial risk for psychosis as the reference group. $N=140-141$ 
Supplementary Table 4. Results of linear regression analyses, when predicting absolute and relative motion by polygenic risk score for schizophrenia.

\begin{tabular}{llllllll}
\hline & \multicolumn{3}{c}{ Absolute motion } & & \multicolumn{3}{c}{ Relative motion } \\
\cline { 2 - 3 } \cline { 6 - 7 } & Beta & $\mathrm{B}$ & $p$ & & Beta & $\mathrm{B}$ & $p$ \\
\hline Age & -0.073 & -0.029 & 0.379 & & 0.048 & 0.002 & 0.568 \\
Sex $^{1}$ & -0.156 & -0.095 & 0.064 & & -0.104 & -0.005 & 0.217 \\
Educational level & 0.051 & 0.031 & 0.545 & & -0.007 & 0.000 & 0.931 \\
Polygenic risk score & -0.064 & -0.020 & 0.439 & & -0.074 & -0.002 & 0.375 \\
$\quad$ for schizophrenia & & & & & & \\
\hline
\end{tabular}

${ }^{1}$ Male as the reference group. $N=148-149$ 